(Ohtsuka Y, Ohtahara S et al. Treatment of intractable childhood epilepsy with high-dose valproate. Epilepsia Jan/Feb 1992; $\underline{33}$ :158-164). (Reprints: Dr. Y. Ohtsuka, Department of Child Neurology, Okayama University Medical School, 2-5-1, Shikata-cho, Okayama 700 , Japan.)

COMMENT. The authors recommend high dose VPA therapy for intractable childhood epilepsy of generalized and partial types and particularly for patients with West syndrome. The monitoring of platelet counts and fibrinogen levels in addition to liver function tests is advised.

Kreuz W et al. from Frankfort, Germany report the induction of von Willebrand disease type $I$ in $67 \%$ of 30 children receiving VPA therapy (Epilepsia Jan/Feb 1991; 33:178-184). The decrease in coagulation parameters were not dependent on either VPA dose or the period of drug administration. This increased tendency to hemorrhage in patients receiving VPA must be considered, especially during surgical intervention and after traumatic events.

Kondo $T$ et al. from Hirosaki University, Japan, report that 3 risk factors (young age, polypharmacy, and high VPA serum level) enhance the susceptibility to VPA hepatotoxicity by altering the metabolism of VPA and by increasing the conversion of VPA to 4-en, the most toxic VPA metabolite (Epilepsia Jan/Feb 1992; $\underline{33}$ :172-177).

\title{
CARBAMAZEPINE-INDUCED DIARRHEA
}

Intractable diarrhea induced by carbamazepine (CBZ) in 3 patients and necessitating discontinuation of the drug is reported from the Departments of Neurology and Medicine, University of Louisville School of Medicine, Kentucky. A 3 year old boy with generalized tonic-clonic seizures received CBZ $125 \mathrm{mg} / \mathrm{day}$, increasing to $125 \mathrm{mg} 3$ times daily. The CBZ serum level was $7.4 \mathrm{mcg} / \mathrm{ml}$. Frequent diarrhea was associated with negative stool examination and cultures. Liver function studies showed minimal LDH elevation. The gradual withdrawal of $\mathrm{CBZ}$ and substitution of phenytoin were followed promptly by recovery. The 2 other patients were adults, both of whom recovered after CBZ was withdrawn and phenytoin or primidone was substituted (Iyer V, Holmes JW, Richardson RL. Intractable diarrhea from carbamazepine. Epilepsia Jan/Feb 1992; 33:185-187). (Reprints: Dr. V. Iyer, Department of Neurology, University of Louisville, Louisville, KY 40292.)

COMMENT. The authors note that diarrhea is described as a complication of CBZ in the package insert but gastrointestinal adverse effects of $\mathrm{CBZ}$ have received little attention in the literature. Other GI complications include anorexia, nausea, vomiting and constipation. Hepatic toxicity has been reported in $5 \%-10 \%$ of patients.

Carbamazepine-induced eosinophilic colitis with severe watery diarrhea is reported in an adult from Helsinki, Finland (Anttila VJ, Valtonen M. Epilepsia Jan/Feb 1992; 33:119-121). With 2 reports of 
CBZ-induced diarrhea appearing in the same issue of Epilepsia, the gastrointestinal side effects of CBZ need to be taken seriously and treated by prompt discontinuation of CBZ.

\section{CEREBRAL PALSY}

\section{NEURODEVELOPMENT AND SERUM BILIRUBIN IN PRETERM INFANTS}

The interrelationships of maximal total serum bilirubin concentrations, neonatal cranial ultrasonographic abnormalities, and the occurrence of cerebral palsy and developmental delay were studied in 249 preterm infants of less than 34 weeks gestation at the Departments of Pediatrics, Neurology, and Radiology, Thomas Jefferson University Hospital, Philadelphia, PA. Of 45 children with spastic cerebral palsy, all but 7 had grade III/IV intracranial hemorrhage or moderate to severe periventricular echodensity or both, and ultrasonographic abnormalities. There was no evidence that bilirubinemia $(2.3$ to $22.5 \mathrm{mg} / 100 \mathrm{~mL}$ total serum bilirubin) was causally related to cerebral palsy, early developmental delay, or periventricular cysts. Large ventricular cysts, moderate to severe periventricular echodensity, and the need for assisted ventilation were associated with the occurrence of cerebral palsy at a statistically significant level (Graziani LJ et al. Neurodevelopment of preterm infants: neonatal neurosonographic and serum bilirubin studies. Pediatrics Feb 1992; $\underline{89}: 229-234)$. (Reprints: Dr. Graziani, Department of Pediatrics, Jefferson Medical College, Thomas Jefferson University, Philadelphia, PA 19107.)

COMMENT. The putative neurotoxic effects of serum bilirubin in the development of spastic cerebral palsy were not supported by this study of preterm infants with neurosonographic abnormalities. The authors note that the significant association between the need for mechanical ventilation and increased risk for $\mathrm{CP}$ in preterm infants requires further study.

The effects of neonatal bilirubin exposure on psychoeducational outcome have been studied in a group of grade school children, 9-11 years old, who required neonatal intensive care at the Department of Pediatrics, University of California, Davis, Sacramento, CA. (Hansen RL. J Dev Behav Pediatr Oct 1991; 12:287-293) A measure of bilirubin binding, calculated directly from the albumin concentration, correlated significantly with the Kaufman Mental Processing Composite Measure of Psychoeducational Outcome but other more direct measures of bilirubin exposure (maximum total serum bilirubin) did not. It is suggested that the calculated albumin - determined binding value may be more appropriate than the total serum bilirubin in assessing clinical management. 